\title{
Corela
}

Cognition, représentation, langage

HS-8 | 2010

L'interpellation

\section{L'interpellation pragmatique dans les articles de commentaire}

\section{Thierry Guilbert}

\section{(2) OpenEdition}

\section{Journals}

Édition électronique

URL : http://journals.openedition.org/corela/1850

DOI : $10.4000 /$ corela. 1850

ISSN : $1638-573 \mathrm{X}$

\section{Éditeur}

Cercle linguistique du Centre et de I'Ouest - CerLICO

Référence électronique

Thierry Guilbert, «L'interpellation pragmatique dans les articles de commentaire », Corela [En ligne], HS-8 | 2010, mis en ligne le 24 novembre 2010, consulté le 19 avril 2019. URL : http:// journals.openedition.org/corela/1850; DOI : 10.4000/corela.1850

Ce document a été généré automatiquement le 19 avril 2019

\section{(c) (i) (2)(2)}

Corela - cognition, représentation, langage est mis à disposition selon les termes de la licence Creative Commons Attribution - Pas d'Utilisation Commerciale - Partage dans les Mêmes Conditions 4.0 International. 


\title{
L'interpellation pragmatique dans les articles de commentaire
}

\author{
Thierry Guilbert
}

\section{Introduction}

1 Nous aborderons ici l'interpellation d'un point de vue élargi en nous appuyant notamment sur le sens que lui donne la philosophie politique d'Althusser. Notre objectif est de proposer l'éclairage (peut-être) inhabituel, celui de l'Analyse des discours, d'une notion plus familière aux grammairiens et linguistes. Cet objectif est lié à notre conception de la notion et nous voudrions, avant toute chose, mettre en garde le lecteur en définissant rapidement ce que nous entendons par interpellation. Tout d'abord, la notion d'interpellation telle que nous la concevons ici ne possède pas le caractère intempestif qu'on lui attribue souvent. Ensuite nous distinguons deux formes d'interpellation différentes ${ }^{1}$ : d'une part, les interpellations que la grammaire et la linguistique identifient sous les vocables d'interjections, apostrophes, exclamations, vocatifs, etc., c'est-à-dire les interpellations lexicalement fixées et reconnaissables et que nous ne prendrons pas en compte ici, et d'autre part, les interpellations que, pour le moment et à défaut d'une meilleure dénomination, nous qualifierons d'interpellations implicites - par exemple «les sans chômage » ou encore «les grèves à la française ", pour ne prendre que des nominations - et que nous tenterons de mieux identifier dans cet article.L'interpellation implicite, qui est certes un fait linguistique, est également un fait discursif et, si elle est marquée en discours, elle n'est pas forcément remarquée au premier abord; autrement dit, pour paraphraser la définition de Kerbrat-Orecchioni $(1986,21)$, elle ne constitue pas « en principe le véritable objet du dire ». La difficulté à saisir l'implicite est parfaitement rendue par l'ambiguïté de ce «en principe» - l'interpellation implicite est-elle ou non le véritable objet du dire? Enfin, l'implicite dans l'interpellation pose d'emblée la question pertinente de la manipulation du dire par le dit: si l'interpellation implicite n'est pas en principe le trait sémantique principal de l'énoncé, nous verrons qu'elle l'est souvent en discours; nous nous demanderons alors quel rôle elle joue dans la présentation évidente des discours idéologiques².Bien que l'on 
puisse nous faire remarquer, à juste raison, que toute interpellation a un aspect ou un niveau pragmatique, c'est en ce sens que notre titre fait mention de l'interpellation pragmatique.

\section{L'interpellation et la problématique de l'évidence}

2 Telle qu'elle est conçue ici, l'interpellation s'inscrit dans ce qu'on pourrait appeler une épistémologie de l'existence. Nous entendons par cette formule que l'interpellation peut être considérée comme le parangon des interrogations individuelles et collectives sur l'existence, que l'interpellation est un mode d'accès au sens du monde ; on retrouve cette signification dans l'expression populaire (qui n'est pas tout à fait passée de mode) : « ça m'interpelle "; le pronom "ça » désignant un paysage, aussi bien qu'une œuvre, une idée... Par cette expression, le locuteur qualifie son mode de perception et son mode de relation à (un fragment de) la réalité. Cette qualification, saturée d'émotion, est également teintée d'une interrogation qui s'adresse justement à notre mode de perception de la réalité : comment ce paysage, cette œuvre, cette idée peuvent-ils me toucher à ce point? Interrogation à laquelle on peut ajouter cette autre qui résulte d'un retournement épistémologique présent dans "ça m'interpelle » et plus généralement dans l'interpellation : comment ce paysage, cette œuvre, cette idée parviennent-ils à me questionner?

3 Ces questions philosophiques ne seront bien sûr pas résolues ni mêmes traitées ici : notre propos se situe ailleurs. Mais puisqu'on a soulevé ces questionnements, qu'on nous permette simplement de postuler que ce mode de perception de la réalité qui se dit ou se confie à l'autre passe par le truchement des interrelations humaines, elles-mêmes structurées et modifiées par les interactions langagières. On peut ainsi concevoir la notion d'interpellation de façon plus large comme la problématique d'une interrelation active tout à la fois entre l'individu et le monde et l'individu et la collectivité. C'est en ce sens que le «ça m'interpelle» et, plus largement, l'interpellation désignent ce questionnement épistémologique sur l'existence auquel nous sommes soumis.

\subsection{L'interpellation althussérienne}

Notre conception s'appuie en partie sur la notion d'interpellation telle qu'elle a été définie par Althusser (1976). La "double interpellation spéculaire du sujet » n'est pas, comme on le pense souvent, la simple imposition ni même la simple soumission à une idéologie (dominante), mais une relation dialectique entre le sujet et cette idéologie : un processus complexe de soumission consentie. L'interpellation est le processus même de l'idéologie, définie comme « une représentation du rapport imaginaire des individus à leurs conditions réelles d'existence» $(1976,101)$. Althusser considère ce qu'on pourrait appeler le processus interpellatif comme une libre adhésion du sujet, en jouant d'ailleurs sur la polysémie du mot « sujet » à la fois l'être libre pourvu de sa subjectivité et l'être assujetti : « l'individu est interpellé en sujet (libre) pour qu'il se soumette aux ordres du Sujet, donc pour qu'il accepte (librement) son assujettissement » (Althusser 1976, 121).

5 Cette liberté de soumission est ainsi toute relative puisque les individus sont « toujoursdéjà des sujets » $(1976,115)$ :

«l'idéologie [...] fonctionne de telle sorte qu'elle «recrute» des sujets parmi les individus (elle les recrute tous), ou transforme les individus en sujets (elle les 
transforme tous) par cette opération précise que nous appelons l'interpellation » $(1976,113)$.

6 La signification que l'auteur donne au verbe « interpeller » est proche du sens des verbes «appeler» et «héler » et de son sens étymologique «interrompre en adressant la parole ». Le langage est d'ailleurs, pour Althusser, l'une des pratiques par laquelle l'idéologie s'adresse aux individus; en d'autres termes, l'interpellation est une forme de questionnement par le langage, lequel, nous l'avons postulé supra, structure notre perception de la réalité.

7 Un dernier point, essentiel pour Althusser: les sujets n'ont pas conscience d'être interpellés. La réalité de cet assujettissement librement consenti est, selon ses propres termes, "nécessairement méconnue ». Pour chacun d'entre nous, la « catégorie de sujet est une "évidence" » (1976, 111); d'ailleurs l'idéologie - et donc l'interpellation fonctionnent "à l'évidence». L'idéologie nous interpelle tous, sans que nous en ayons pleinement conscience. Il s'agit donc d'un processus implicite que nous acceptons librement sans que notre avis ait été sollicité en quelque manière. Ajoutons que cette soumission consentie et évidente n'est pas exempte de violence et s'inscrit bien sûr dans le cadre des luttes entre les différentes classes sociales ${ }^{3}$. C'est ce phénomène en apparence paradoxal que nous étudierons ici.

\subsection{Bourdieu et la reconnaissance}

8 Cette question de la libre soumission est traitée également par Bourdieu (1982). Tout en développant les notions de reconnaissance, d'imposition symbolique et d'habitus (1979, 1982), Bourdieu, d'une façon analogue, propose cette idée d'une participation de l'individu qui « défie l'alternative ordinaire de la liberté et de la contrainte » $(1982,36)$. Ainsi l'imposition symbolique est une reconnaissance qui n'est ni « soumission passive », ni « libre adhésion ». L'imposition symbolique, proche de l'interpellation althussérienne, est un «travail d'objectivation» qui a pour conséquence l'acceptation des valeurs dominantes. L'interpellation est donc en quelque sorte, au sens sociologique et sociolinguistique, un processus de réinterprétation qui se fait au prix de «l'unification des opposés » et de la "dénégation des divisions» $(1982,18)$ et qui rappelle ce que Durkheim appelle le « consensus rationnellement accepté » ou encore « la reconnaissance rationnelle de la nécessité d'une cohésion sociale » (1895; cité par Péquignot et Tripier 2000, 133), consensus qui n'est d'ailleurs pas considéré par l'auteur comme un choix entièrement libre ${ }^{4}$. En effet, dans quelle mesure ce qui peut apparaître comme « rationnellement accepté » est-il un choix véritablement libre? On aurait tort ici d'occulter la « violence symbolique » des discours dominants (Bourdieu 1982).

\subsection{Le schéma participatif de l'interpellation}

9 On peut également ajouter que l'interpellation s'inscrit dans une «configuration interpellative » (Détrie 2006), c'est-à-dire dans la problématique d'un schéma participatif et hiérarchisé entre l'individu et le monde social. Le locuteur provoque, en adressant la parole à l'interlocuteur, une certaine proximité et une forme de coopération. S'il y a bien une «contextualisation", c'est-à-dire une re-construction par les participants des données pertinentes du contexte dans l'interaction (Schegloff 1992), il y a plus encore la co-énonciation d'un texte comme étant le produit de cette interaction. Toutefois, cette 
action volontaire de participation entre les interactants est asymétrique car l'interpellé n'a ni l'initiative, ni le choix de la situation de communication: le consentement de l'interpellé n'est ni « soumission passive », ni « libre adhésion » à celui qui l'interpelle. Il y aurait donc une indéniable visée pragmatique dans ce processus : produire l'évidence d'une coopération, c'est-à-dire produire l'évidence d'un consensus.

Ce processus d'interpellation, tel que nous venons de le préciser à travers divers auteurs, et dont on peut postuler qu'il est un processus discursif lui-même inscrit dans une interrelation co-énonciative (c'est-à-dire un contexte dialogal mais également dialogique), situe clairement notre problématique dans une approche socio-pragmatique de l'analyse du discours: il s'agit ainsi de montrer en quoi l'interpellation de nature implicite peut être un acte illocutoire indirect. On s'intéresse ainsi au fonctionnement discursif de l'effet d'évidence idéologique notamment dans le(s) processus d'interpellation utilisé(s) par les articles de commentaire ${ }^{5}$.

\section{L'interpellation pragmatique}

\subsection{Le corpus}

11 Notre corpus est composé de 184 éditoriaux et chroniques issus de dix publications de la presse française lors de deux crises sociales (novembre-décembre 1995 et mai-juin 2003), de deux « moments discursifs » (Moirand 2002) : parce que les prises de position en temps de crise nous semblaient plus repérables que d'ordinaire, nous avons choisi ces deux moments de crise pour leur comparabilité thématique - deux mouvements sociaux s'opposant à des modifications des régimes de retraites des fonctionnaires et apparentés ${ }^{6}$.

12 Nous avons choisi le genre article de commentaire parce qu'il est pourvu de l'autorité énonciative et du droit de donner sens à l'actualité. L'analyse du corpus montre que le genre "article de commentaire» peut utiliser l'interpellation dans le but (persuasif) d'imposer une perception idéologique de l'événement, c'est-à-dire dans le but d'en constituer une représentation partagée. Le processus d'interpellation implicite - dont nous ne pouvons définir les formes à l'avance- par l'article de commentaire comporterait, selon nous, trois niveaux.

\subsection{La didacticité au service de l'interpellation}

Le premier niveau étudié est didactique. En effet, l'article de commentaire est un texte argumentatif qui apporte de nouveaux savoirs de manière implicite(Petiot 1992; Poli 1992) : le journaliste détient un savoir dont il pense que son lecteur en est dépourvu. Pourtant, l'article de commentaire n'a pas pour vocation première la transmission des connaissances : c'est un « discours-limite de transmission des connaissances » (Beacco et Moirand 1995, 34). La didacticité de l'article de commentaire reste au service des objectifs argumentatifs et pragmatiques du journaliste qui se pose en tant que médiateur entre le savoir et le lecteur. La situation de communication est donc asymétrique, l'éditorialiste ou le chroniqueur interpelle le lecteur en lui présentant prioritairement, sous forme évidente, les informations qui sont nécessaires au déploiement de son argumentation.

$14 \mathrm{Si}$, au début de l'extrait suivant, le chroniqueur commence par « rappeler les faits » de façon implicite (par la présupposition «système de retraites condamné»), dans les 
deuxième et troisième paragraphes, il se veut pédagogue, cette fois de façon explicite, en faisant appel tout d'abord à l'autorité symbolique d'un écrit philosophique puis à celle du dictionnaire Littré :

Le Figaro, 12/05/03, « La France cassée », Alain-Gérard Slama:

«En ce mois de mai inactif, dans l'attente des vacances prochaines, et aux approches d'une grève des syndicats acharnés à défendre un système de retraites condamné, la France est dans un état qu'on hésite à nommer: asthénique? régressif ? éclaté ? moisi ? On cherche le mot.

Il nous est donné par le titre d'une pièce oubliée du philosophe Gabriel Marcel, qui fut créée en 1932, dans une période de montée des périls comparable à la nôtre : Le Monde cassé. On ne saurait mieux dire. [...]

Littré donne, du participe cassé, les définitions suivantes: 1) Mis en deux ou plusieurs fragments. Un bras cassé. 2) Annulé. Un arrêt cassé par la cour supérieure. 3) Renvoyé de son emploi, de son grade. 4) Affaibli par l'âge. Cassé de vieillesse. 5) Voix usée, qui se fait à peine entendre. L'article de Littré s'achève sur l'expression proverbiale : "Il en paiera les pots cassés." En effet. »

15 Il s'agit à travers l'explicitation et la justification du choix du titre de la chronique de persuader le lecteur de l'évidence du constat. À chaque recours explicite à la didacticité correspond un court commentaire métadiscursif (« On ne saurait mieux dire.» et «En effet. ») qui renvoie directement au constat implicite de la présupposition relevée dans le premier paragraphe. Or celle-ci interpelle le lecteur par le truchement du déjà-dit et du déjà-entendu.

En effet, la didacticité ne consiste pas à apporter uniquement des savoirs nouveaux mais peut, au contraire, activer des connaissances partagées ou présentées comme telles :

Le Nouvel Observateur, 22/05/03, Laurent Joffrin :

«Les rituels saisonniers de la grève à la française font la joie de la presse anglo-

saxonne et des touristes japonais. »

Il s'agit ici de la première phrase du texte. La partie soulignée est une nominalisation axiologique et ironique : la nominalisation « les rituels saisonniers de la grève » fait appel (interpelle) aux connaissances partagées par le scripteur et le lecteur en évoquant des grèves passées (mai 68 par exemple) ; «à la française » produit une «folklorisation » du mouvement en inscrivant celui-ci dans la tradition nationale au même titre que la gastronomie, la tour Eiffel ou encore la baguette de pain. Le syntagme nominal dans son ensemble, « les rituels saisonniers de la grève à la française ", produit donc la réification de ce mouvement et, plus généralement, des mouvements d'opposition; conséquence : les revendications propres à ce mouvement précis sont occultées. L'effet d'évidence de cette interpellation est le résultat à la fois de la didacticité de la nominalisation - qui comporte une présupposition d'existence et a un effet didactique d'activation de connaissances partagées - et de l'aspect constatif de l'énoncé.

Libération, 13/12/95, Guillaume Malaurie :

"La vaste rébellion qui s'exprime aujourd'hui est bien plus grave, plus profonde, que ne le traduisent avec des mots surannés les dirigeants syndicaux. »

Ce type de désignation (Petit 2002) attribue de façon implicite un sens à l'événement: l'effet d'évidence vient à nouveau de la présupposition d'existence, renforcée par le déictique « aujourd'hui » qui affirme la forme constative, il ancre le constat dans l'ici et maintenant.

L'Express, 05/06/03, Denis Jeambar :

"[...] les sans-culottes se dressaient contre l'absolutisme; les "sans chômage" qui prennent aujourd'hui le pays en otage par des grèves à répétition contestent en fait 
un pouvoir démocratiquement élu, c'est-à-dire la principale conquête de la Révolution. »

19 Nous avons affaire ici à ce que Siblot (2001) appelle une nomination. "Les "sans chômage" » renvoie une fois encore à des connaissances présentées comme partagées : le lecteur saisit que l'éditorialiste fait référence aux fonctionnaires dont le statut ne leur fait pas craindre le chômage. Ici aussi, la présupposition d'existence, incluse dans la relative / il y a des " sans chômage » qui prennent le pays en otage/, est renforcée par le déictique " aujourd'hui » et son aspect constatif. De plus, la symétrie temporelle et historique de la phrase appelle à la comparaison des éléments. Ainsi la nomination « les "sans chômage" » assimile implicitement les fonctionnaires aux privilégiés de l'Ancien régime tout en les frappant littéralement d'excommunication démocratique: le journaliste leur dénie implicitement le droit à s'opposer au pouvoir.

\subsection{L'interpellation énonciative}

Le second plan de l'interpellation implicite est énonciatif. L'éditorialiste et le chroniqueur associent le destinataire à leur dire par un "nous" inclusif: c'est une loi du genre " article de commentaire "; l'intérêt du "nous » inclusif, mais aussi du "on » indéfini (qui prend la plupart du temps la même valeur), est de partager la responsabilité du propos avec le lecteur, de le co-responsabiliser. C'est que nous appelons la " désubjectivisation " de l'instance énonciative. Cette désubjectivisation n'est bien sûr qu'apparente. L'éditorialiste et le chroniqueur semblent inviter le destinataire à participer à l'élaboration du dire. En ce sens, par l'interpellation énonciative, le destinataire de l'article de commentaire est présent par le dire dans ce qui est dit : l'interaction prend bien la forme d'un schéma participatif, la forme de la co-énonciation évoquée ci-dessus.

Le Nouvel Observateur, 29/05/2003, Jacques Julliard :

" On avait parlé en 1995 de grèves par procuration. Nous assistons aujourd'hui à des

grèves par provision. »

21 Notons au passage que, dans cette mise en perspective de la première phrase, on retrouve une marque de didacticité. Le «nous » de la seconde phrase englobe l'auteur et le lecteur dans une même vision du monde. Mais, plus encore qu'une co-énonciation ou même une " contextualisation " (voir supra 1.4), le propos assumé par le «nous» inclusif est une socialisation fallacieuse de l'énonciation, car cette forme énonciative reste le produit de l'asymétrie d'un «Je dilaté » (Benveniste 1966). La désubjectivisation énonciative bénéficie ainsi de l'autorité de la voix collective et agit comme une modalité véridictoire, elle est renforcée à nouveau par le déictique « aujourd'hui » qui permet d'ancrer l'énoncé dans l'ici et maintenant et de renvoyer à une « réalité » qui, en retour, semble valider cette vision de l'événement.

La désubjectivisation énonciative, tout comme les nominalisations, permet donc de présenter une opinion ou un positionnement idéologique particuliers sous la forme d'une interpellation, c'est-à-dire sous la forme d'un appel à des connaissances toujours-déjà partagées; en dernier instance, ces procédés sont à même de constituer de nouveauxsavoirs ou de transformer des positions particulières en doxa. Cette activation de connaissances toujours-déjà partagées ou de connaissances préexistantes ressemble fort à ce que M-A Paveau $(2007,102)$ appelle "prédiscours » : « un cadre prédiscursif collectif contribuant à l'élaboration des discours en activant un ensemble de savoirs, croyances et pratiques ». Toutefois, ce qui se présente ici comme des prédiscours, comme activation de 
connaissances toujours déjà partagées, n'est que scénographie fallacieuse, n'est que manipulation discursive. En effet, ce qui se présente comme activation de connaissances partagées ou activation d'un sens commun, ce qui utilise à la fois la force d'interpellation des prédiscours et l'autorité énonciative de la voix collective, a pour visée de produire cette impression de reconnaissance présente dans l'interpellation et, par là même, l'effet d'évidence :

Le Point, 02/05/2003, Claude Imbert:

"Le dépensier majeur, c'est, on le sait, l'Etat employeur »

Le Monde, 27/05/03, Jean-Marie Colombani :

«[...] enfin, une opinion convaincue de la nécessité de la réforme. »

Paris-Normandie,12/05 /2003, Gilles Dauxerre :

" [...] chacun s'accorde, sur la nécessité d'une réforme et d'un effort partagé »

Ici encore, les désubjectivisations, en position de sujet ou sous forme d'incises, activent des connaissances présentées comme partagées et préexistantes. Elles visent à produire une nouvelle doxa ; elles se doublent - nous anticipons sur le niveau pragmatique - de tropes illocutoires. Il y a ici constitution de deux topiques néolibérales :

/il faut réduire les dépenses de l'Etat/ (antienne du Point)

/il faut accepter la nécessité de la réforme (de l'État)/ (Le Monde, Paris-Normandie)

On peut citer également les questions rhétoriques (largement utilisées par les discours didactiques) comme autre forme énonciative de l'interpellation implicite : à l'instar des désubjectivisations, elles ne sont pas directement prises en charge par l'énonciateur et produisent également cet effet d'évidence de l'interpellation en fixant, comme les présuppositions, un « cadre contraignant » à la communication (Ducrot 1984).

Le Nouvel Observateur, 05/06/03, Laurent Joffrin :

« Tous ceux-là, CGT comprise, sont-ils des complices du patronat? Des agents de la mondialisation ultralibérale? Des traîtres à la classe ouvrière?»

La réponse à une question rhétorique étant évidente, l'essentiel réside bien sûr dans l'implicite de la question elle-même. Dans cet extrait, la succession de questions rhétoriques désubjectivisées vise à stigmatiser ironiquement les opposants, en faisant de ceux-ci les locuteurs virtuels d'un lexique «stalinien»(«complices», «agents », «traîtres ») et en les amalgamant à ceux qu'« on »(la doxa) considérerait comme les plus dogmatiques et archaïques des communistes. Dans cet exemple, le commentaire non pris en charge par le journaliste a donc pour effet d'ostraciser les adversaires aux projets gouvernementaux, tout en activant des représentations (censées) partagées, et de produire une nouvelle doxa: l'événement décrit n'est qu'une réplique de luttes (dé)passées et archaïques, réplique affaiblie de bouleversements sociaux caducs et moribonds.

Le Figaro, 12/05/03, Alain-Gérard Slama :

«En ce mois de mai inactif, dans l'attente des vacances prochaines, et aux approches d'une grève des syndicats acharnés à défendre un système de retraites condamné, la France est dans un état qu'on hésite à nommer : asthénique ? régressif ? éclaté ? moisi ? On cherche le mot.

[...] Littré donne, du participe cassé, les définitions suivantes : [...]. L'article de Littré s'achève sur l'expression proverbiale : "Il en paiera les pots cassés." En effet. »

Nous reprenons cet exemple déjà utilisé (voir 2.1) car il permet de montrer combien les deux premiers niveaux, à savoir les niveaux didactiques et énonciatifs, interviennent de façon complémentaire dans l'argumentation par l'interpellation implicite. Le second niveau venant au secours du premier en naturalisant de l'aspect idéologique du discours. L'aspect fortement didactique donné à l'extrait cautionne la recherche du mot adéquat ; 
pourtant, non seulement le propos est chargé d'un trope présuppositionnel (KerbratOrecchioni 1986) - puisque la présupposition, présentée comme un savoir partagé (/le système de retraites est condamné/), constitue en discours le véritable objet du dire -, mais le journaliste met en scène son travail de scripteur recherchant le mot juste tout en associant le destinataire à son dire par le «on » (« on hésite à nommer », " on cherche le mot» ne sont pas univoques), ce qui lui permet, par l'énumération, d'attribuer les qualificatifs "asthénique ", "régressif ", " éclaté ", «moisi ", pour le moins orientés, à «l'état de la France ». Le lecteur est donc doublement interpellé de façon didactique et énonciative, mais cette interpellation reste implicite, de sorte que le constat s'impose comme une évidence.

La question rhétorique (Kerbrat-Orecchioni 1986), la présupposition (Ducrot 1984) et la nomination (Siblot 2001) sont donc des modalités énonciatives de l'interpellation par la désubjectivisation, l'implicite et l'activation de connaissances présentées comme partagées. Elles participent d'une mise en scène co-énonciative et consensuelle dont le lecteur serait partie prenante: elles masquent l'asymétrie interpellative pourtant constante, voire constitutive de l'article de commentaire. Rappelons cependant que nous considérons que l'acte pragmatique interpellatif n'est pas circonscrit à ces quelques formes linguistiques ou discursives précises, celles-ci ou d'autres, et qu'il peut s'actualiser dans des formes plus larges encore.

\subsection{L'interpellation pragmatique et communicationnelle}

Le troisième niveau est donc pragmatique et communicationnel. L'éditorialiste (ou le chroniqueur) interpelle également le lecteur selon divers procédés qui, tous, ont pour fonction de référer, explicitement ou non, à des schématisations, à des catégorisations ou encore à des cadres doxaux préexistants ou présentés comme tels. Il s'agit non seulement d'activer les connaissances partagées, la doxa, mais aussi de constituer en doxa les opinions personnelles du journaliste, opinions présentées comme étant doxiques. Ainsi, les procédés communicationnels et argumentatifs tels que l'emploi de formes parémiques (Tamba 2000), l'activation de représentations partagées (comme «la comparaison au voisin ", «le bon père de famille » ou «la guerre des chefs») et la généralisation de positionnements individuels par la généricité du discours (Guilbert 2007), l'utilisation de la figure de l'opinion publique (Landowski 1989), ou encore la présentation de l'événement sous la forme d'un « cadre naturel »(Goffman 1981) sont autant de rappels à la norme et d'injonctions implicites, et quelquefois explicites, à caractère interpellatif.

Du point de vue communicationnel, si la situation est co-construite par les participants mais elle l'est également régie par les structures sociales (Fairclough, 1995; van Dijk, 2006) qui agissent sur le discours de façon indirecte par l'intermédiaire d'une interface cognitive :

«les contextes ne devraient pas être définis comme une sorte de situation sociale dans laquelle le discours prend place, mais plutôt comme une représentation sociale, ou comme un modèle construit par les locuteurs à propos d'une telle situation » (van Dijk 2006, 84).

Ainsi, il nous semble que, même si ces points de vue épistémologiques diffèrent quelque peu de ceux d'Althusser ${ }^{7}$, ces remarques peuvent être utiles pour comprendre le processus interpellatif puisque, nous l'avons remarqué, le journaliste active les représentations socialement partagées. L'interface cognitive, grâce à laquelle se 
construisent les modèles de perception d'une situation ou un événement, permet de préciser le processus par lequel sont déterminées les représentations sociales. Nous avons insisté sur l'activation de connaissances partagées aux niveaux didactique et énonciatif dans le processus de l'interpellation implicite, le niveau pragmatique et communicationnel supposerait l'activation de modèles préexistants; cette activation se remarque, par exemple, dans le détournement de proverbes et plus largement dans le détournement d'énoncés parémiques :

Le Point, 11/11/95, Claude Imbert :

"Qui, pour finir, décidera du succès ou de l'échec ? Ce gouvernement remanié prêt à en

découdre? Ou bien le citoyen inconséquent qui voudra le beurre et l'argent du beurre

?»

31 La dernière question rhétorique, fortement axiologique («citoyen inconséquent »), comprend un élément d'énoncé parémique (ici un dicton) qui vise à légitimer l'orientation éditoriale du journal et à stigmatiser le citoyen qui, malgré l'interpellation implicite par un modèle doxique, persisterait dans son inconséquence irréaliste à ne pas reconnaître la justesse des propositions gouvernementales. Cette question est un trope illocutoire (Kerbrat-Orecchioni 1986), la valeur pragmatique patente ne coïncide pas avec la valeur pragmatique latente de l'énoncé: cette fausse question est une véritable injonction (prescription), cette valeur pragmatique est d'ailleurs celle du dicton luimême.

L'Express, 30/11/95, Christine Ockrent :

"Le temps, c'est de l'argent, et l'argent coûte trop cher.»

Ici l'adage garde sa valeur pragmatique habituelle de prescription: /ne perdons pas de temps/. La seconde partie de l'énoncé, frappée au coin du "bon sens économique », se présente ainsi comme le prolongement «naturel» de l'adage. Ce qui est présenté ici comme le rappel d'une évidence est pourtant le cadre de perception du marché, un cadre idéologique strictement financier. Cet énoncé prend la forme d'un "énoncé généralisateur ", au sens où nous l'entendons (Guilbert 2007, 199-202), c'est-à-dire qu'il comporte une désubjectivisation, un présent gnomique et une modalité déontique (sous forme de trope illocutoire). Cette forme permet à l'éditorialiste d'énoncer comme une loi universelle un point de vue particulier qui résulte de l'analyse d'un événement singulier.

Ces notions de modèle et de catégorisation renvoient également à la notion de « cadre de primaire» de Goffman (1984). Il existerait ainsi deux types de cadres primaires de perception $^{8}$ :les cadres naturels ou non pilotés purement physiques, sans aucune conscience ou intention préalable, et les cadres sociaux ou pilotés qui présupposent une action humaine. Or, nous avons remarqué troiscaractéristiques essentielles des cadres naturels en ce qui concerne l'effet d'évidence de l'interpellation : (i) ils sont naturels donc vraisemblables, (ii) l'absence de cause ou d'intention leur confère un aspect neutre, (iii) leur nature «purement physique» les font apparaître inévitables (Guilbert 2007). Ainsi lorsqu'un journaliste propose un cadre de perception naturel d'un événement pourtant piloté, son discours produit, nous semble-t-il, un effet pragmatique qui oriente la perception de cet événement : le modèle «cadre naturel » interpelle le destinataire en modifiant sa vision de l'événement.

L'Express, 05/06/03, Claude Allègre :

"Plongés dans une mondialisation aveugle et sans régulation, nous subissons les

"humeurs" du temps.»

Dans le cas d'une "énonciation de cadre naturel», trois critères nous semblent nécessairement présents : une désubjectivisation, un élément naturel en position d'actant 
et une modalité véridictoire (Guilbert 2007, 202-204); celle-ci a pour effet d'établir un cadre naturel de perception de la réalité.

Ici, la désubjectivisation prend la forme d'un "nous » qui a la valeur la plus englobante qui soit puisque, dans le cadre de la mondialisation, il s'agit de l'humanité. Par ailleurs, la mondialisation est présentée comme un phénomène non piloté («sans régulation »), naturel et sans intention («aveugle »). Par ailleurs, puisque l'humanité «subit» «les "humeurs" du temps ", cet élément naturel est bien en position d'actant. Enfin, la forme constative et le présent d'énonciation qui ancrent l'énoncé dans l'ici et maintenant marquent la modalité véridictoire. Le cadre de perception fixé ici est bien une interpellation au sens althussérien, puisqu'il propose une mise en perspective qui naturalise une idéologie en décrivant la réalité comme imprévisible, violente et, surtout, inéluctable. Cet énoncé comporte donc un trope pragmatique prescriptif:/il faut s'adapter en se pliant à ces nouvelles conditions qui nous dépassent/.

\section{Conclusion}

L'interpellation pragmatique dans les articles de commentaire agit donc à trois niveaux: les niveaux didactique, énonciatif et pragmatique. Nous avons montré combien ces niveaux sont entremêlés. Par conséquent, la visée argumentative de l'article de commentaire s'apparente plus à la persuasion par l'évidence qu'à la conviction rationnelle, autrement dit plus à l'interpellation althussérienne qu'à la libre adhésion. Le médium contraignant qu'est l'article de commentaire est un bon exemple d'interpellation pragmatique en ce qu'il impose une perception à la fois idéologique et évidente de l'événement - c'est là l'aspect dialectique de l'interpellation - par l'activation de la doxa et de connaissances présentées comme partagées, la co-responsabilisation du dire et l'appel aux cadres sociaux de perception. La visée pragmatique de l'article de commentaire repose ainsi sur l'interpellation implicite, c'est-à-dire sur l'impression de reconnaissance vis-à-vis de ce qui est présenté comme socialement partagé par le journaliste et le lecteur, de ce qui est présenté comme une co-énonciation consensuelle. Interpellé par ce qui prend la forme énonciative d'un point de vue qu'il partage, le lecteur n'a plus alors qu'à se rendre à l'évidence.

\section{BIBLIOGRAPHIE}

Althusser, L. (1976). Idéologie et appareils idéologiques d'État. Positions (pp. 67-125). Paris : Editions sociales.

Bakhtine, M. (1977). Marxisme et philosophie du langage. Paris : Minuit.

Beacco, J.-C., \& Moirand, S. (1995). Autour des discours de transmission de connaissances.

Langages, 117, pp. 32-53.

Benveniste, E. (1966). Problèmes de linguistique générale. Paris : Tel Gallimard. 
Bourdieu, P. (1982). Ce que parler veut dire. Paris : Fayard.

Détrie, C. (2006). De la non-personne à la personne : l'apostrophe nominale. Paris : CNRS Editions.

Ducrot, O. (1984). Le dire et le dit. Paris : Minuit.

Fairclough, N. (1995). Critical Discourse Analysis: The Critical Study of Language. London : Longman Group Limited.

Goffman, E. (1984). Les cadres de l'expérience. Paris : Minuit.

Guilbert, T. (2007). Le discours idéologique ou la force de l'évidence. Paris : L'Harmattan.

Kerbrat-Orecchioni, C. (1986). L'implicite (éd. 1998). Paris : Armand Colin.

Landowski, E. (1989). La société réfléchie. Paris : Seuil.

Moirand, S. (2002). Moments discursifs. In Charaudeau P., \& Maingueneau D., Dictionnaire

d'analyse des discours. Paris : Seuil.

Paveau, M.-A. (2006). Les prédiscours. Sens, mémoire, cognition. Paris : Presses de la Sorbonne nouvelle.

Paveau, M.-A. (2007). Les normes perceptives de la linguistique populaire. Langage et société, 119, pp. 93-109.

Péquignot, B., \& Tripier, P. (2000). Les fondements de la sociologie (chap. 8). Paris : Nathan

Université.

Petiot, G. (1992). Néologisme et discours médiatiques. Carnets du Cediscor, n千₹2, pp. 153-165.

Petit, G. (2002). Dénomination/désignation. In Charaudeau P., \& Maingueneau D., Dictionnaire

d'analyse des discours. Paris : Seuil.

Poli, M.-S. (1992). Discours médiatiques de la critique d'art : quand le texte devient le précepteur du lecteur. Carnets du Cediscor,2, pp. 167-175.

Reboul O. (1980). Langage et idéologie. Paris : PUF.

Schegloff, E. (1992). In Another Context. In Durant \& Goodwin (Eds.), Rethinking Context (pp. 191-229). Cambridge: CUP.

Siblot, P. (2001). De la dénomination à la nomination : Les dynamiques de la signifiance nominale et le propre du nom. Cahiers de praxématique, 36, pp. 189-214.

Tamba, I. (2000). Le sens métaphorique argumentatif des proverbes. Cahiers de praxématique, 35, pp. 39-57.

Van Dijk, T. A. (2006). Politique, idéologie et discours. SEMEN, 21, pp. 73-102.

\section{NOTES}

1. Bien sûr les deux types d'interpellation distingués ici ne sont pas diamétralement différents dans leur nature, ils partagent des points communs : fonction phatique, force illocutoire, rapport hiérarchique entre interpellant et interpellé ; cependant leur forme, leur composant linguistique diffèrent sensiblement et c'est ce sur quoi nous basons notre distinction, laquelle implique l'opposition implicite/explicite au centre de notre travail.

2. En ce qui concerne l'évidence des discours idéologiques, voir Reboul (1980) et notre ouvrage Guilbert (2007).

3. Voir l'appendice à l'article cité rédigé par l'auteur. 
4. Voir, par exemple, la définition que Durkheim donne du fait social : «Est fait social toute manière de faire, fixée ou non, susceptible d'exercer sur l'individu une contrainte extérieure.» (Durkheim 1895 Les règles de la méthode sociologique Paris, PUF, 1967, 14; cité in Péquignot \& Tripier 2000, 129)

5. Il nous apparaît justement que l'interpellation implicite, par définition, est multiple et que les différentes formes qu'elle prend ne peuvent être prédites à l'avance, ce qui les distingue des interpellations grammaticales et linguistiques qui peuvent l'être plus aisément. C'est pour cette raison que nous l'abordons sous ses formes pragmatico-énonciatives, et c'est ce que nous entendons par « fonctionnement discursif».

6. Nous évitons l'emploi du mot réforme qui est problématique : l'attribution du sens et des connotations données à ce mot est justement l'un des enjeux idéologiques principaux pour les différents acteurs de ces conflits. Cette attribution détermine la façon dont les citoyens seront interpellés; on voit ainsi combien l'interpellation s'inscrit dans le cadre des «luttes pour l'attribution du sens » (Bakhtine 1977).

7. Bien que dès le début de son ouvrage, Fairclough (1995) revendique explicitement l'influence d'Althusser.

8. Selon Goffman (1984), dans toute situation, surtout lorsqu'elle est nouvelle ou inhabituelle, nous nous posons la question suivante: «que se passe-t-il ici?» Autrement dit, nous recherchons un cadre de perception connu, préexistant qui pourrait s'appliquer à la situation. Il existerait, selon Goffman, un nombre fini de cadres de perception nous permettant d'appréhender l'infinité des situations. Ces cadres sont bien sûr culturels.

\section{RÉSUMÉS}

L'interpellation, considérée ici du point de vue de la réflexion philosophique d'Althusser, est conçue comme un questionnement implicite et dialectique de et sur la réalité et l'existence. Il s'agit de comprendre en quoi l'interpellation permet de présenter un discours idéologique en discours d'évidence, en cela va de soi. L'interpellation à laquelle on s'intéresse n'est pas formelle, mais pragmatique. Ce qui signifie qu'elle peut prendre des formes diverses et inattendues. Ainsi, plutôt que d'étudier le comportement linguistique de certaines formes bien établies, notre choix méthodologique a consisté à étudier l'interpellation selon des points de vue différents - les niveaux didactique, énonciatif et pragmatique - et d'en déduire des appareils plus ou moins formels, autrement dit des procédés interpellatifs. Le corpus étudié se compose d'éditoriaux et chroniques issus de la presse généraliste lors de deux moments de crise sociale en France (1995 et 2003). On verra comment ces articles de commentaire qui ont pour fonction de donner sens à l'actualité utilisent une forme d'interpellation implicite qui a pour effet de naturaliser l'idéologie néolibérale.

Interpellation, regarded here from Althusser's political reflection, is considered as an implied and dialectical question from and about reality and existence. The point is to understand how interpellation helps present an ideological discourse as a discourse of the obvious, as taken for granted. The interpellation we are interested in is not formal, but pragmatic. That is to say it can take various and unexpected forms. Thus, instead of examining the functioning of well-known linguistic forms, our methodological contention is to study interpellation in accordance with different points of view - didactic, enunciative and pragmatic levels - and to infer from such 
analyses formal mechanisms, interpellative processes. The corpus studied is made up of leading articles and columns extracted from the press during two periods of social crisis in France (1995 and 2003). It will be shown how these commentary articles, which purport to give meaning to current events, use an implied interpellation form which leads to the naturalization of the neoliberal ideology.

INDEX

Mots-clés : interpellation, évidence, implicite, idéologie, didacticité

Keywords : interpellation, obviousness, implied utterances, ideology, didactics

AUTEUR

THIERRY GUILBERT

Université de Picardie, CURAPP - UMR 6054 